\title{
Análise da susceptibilidade térmica de ligantes asfálticos através da energia de ativação de fluxo
}

\author{
Raquel Moraes Bringel ${ }^{1}$; Sandra de Aguiar Soares ${ }^{2}$; Jorge Barbosa Soares ${ }^{3}$
}

\begin{abstract}
Resumo: A energia de ativação de fluxo $\left(E_{f}\right)$ é um parâmetro que pode ser relacionado com a susceptibilidade térmica dos ligantes asfálticos puros e modificados, possibilitando informações úteis acerca do desempenho destes em serviço. A obtenção deste parâmetro se dá através da técnica da viscosimetria ou da técnica da reometria. Neste trabalho, os ensaios para o cálculo da $E_{f}$ foram realizados em um viscosímetro rotacional e em um reômetro de cisalhamento dinâmico (DSR). As equações utilizadas nas correlações com cada técnica estão aqui descritas. Os ligantes que apresentaram mais baixos valores da $E_{f}$ são considerados menos susceptíveis às variações de temperatura nas faixas trabalhadas. Os ligantes modificados com o polímero EVA apresentaram $\mathrm{E}_{\mathrm{f}}$ mais baixas e, portanto, menor susceptibilidade térmica do que aqueles que contêm o polímero SBS. Observou-se também que, após o envelhecimento oxidativo, a ordem da susceptibilidade térmica dos ligantes mostrou-se diversa em relação à das amostras não envelhecidas, demonstrando a influência dos polímeros e aditivos no processo de envelhecimento. A presença dos aditivos (cal hidratada e LCC) parece contribuir para reduzir a susceptibilidade térmica do ligante.
\end{abstract}

Abstract: The flow activation energy $\left(E_{f}\right)$ is a parameter which can be related to the thermal susceptibility of pure and modified asphalt binders, providing useful information about their performance in service. This parameter can be obtained using either viscosimetry or rheometry. In this work, experiments for flow activation energy calculation were performed in a rotational viscometer as well as in a Dynamic Shear Rheometer (DSR). Equations used in correlations with each technique are described here. The asphalt binders that showed lower flow activation energy values are considered less susceptible to temperature variation in the temperature ranges studied. The temperature susceptibility of asphalt binders showed that EVA modified asphalt binders have lower degradation than those containing SBS polymer. It was also observed that the oxidative ageing affected the sensitivity of asphalt binders to temperature. Furthermore, after the oxidative ageing, the thermal susceptibility of modified asphalt binders was different, showing that polymers and additives affect in the ageing process. The additives (lime and cashew nut shell liquid) also contributed to the reduction of the thermal susceptibility of asphalt binders.

\section{INTRODUÇÃO}

A adição de polímeros ao asfalto tem mostrado um aumento no desempenho dos pavimentos (Bringel et al., 2005; 2006; Yildirim, 2007). Os polímeros podem modificar a reologia do asfalto em virtude de seus altos pesos moleculares, emaranhado de cadeias e interações de polímero-asfalto (físicas ou químicas) (Jin et al., 2002). Esta modificação pode ser demonstrada por análises viscoelásticas e dinâmicas-mecânicas (Bringel, 2007). A adição de polímeros ao cimento asfáltico de petróleo (CAP), tais como o copolímero de estireno-butadieno-estireno (SBS) (Lucena, 2005; Masson et al., 2005; Bringel et al., 2006) e o copolímero de etileno e acetato de vinila (EVA) (Alencar et al., 2006; 2007), tende a melhorar suas propriedades viscoelásticas proporcionando maior estabilidade ao material do revestimento (Gonzalez et al., 2004).

\footnotetext{
${ }^{1}$ Raquel Moraes Bringel, Universidade Federal do Ceará, Departamento de Química Orgânica e Inorgânica, Fortaleza, CE, Brasil. (e-mail: raquelmmoraes@hotmail.com).

${ }^{2}$ Sandra de Aguiar Soares, Universidade Federal do Ceará, Departamento de Química Orgânica e Inorgânica, Fortaleza, CE, Brasil. (e-mail: sas@ufc.br).

${ }^{3}$ Jorge Barbosa Soares, Universidade Federal do Ceará, Departamento de Engenharia de Transportes, Fortaleza, CE, Brasil. (e-mail: jsoares@det.ufc.br).
}

Manuscrito recebido em 14/7/2008 e aprovado para publicação em $14 / 6 / 2009$. Este artigo é parte de TRANSPORTES, volume XVII, número 1, junho de 2009. ISSN: 1415-7713.
O estudo da reologia dos materiais betuminosos tem por objetivo principal encontrar a relação entre deformação, tensão, tempo de aplicação da carga e temperatura. A investigação dessas propriedades é importante para a compreensão do desempenho do CAP, quando submetido a tensões provenientes do tráfego e a tensões térmicas, uma vez que estes estão sujeitos a grandes variações de temperatura desde o processo de preparação e ao longo da vida útil do pavimento. Várias publicações tratam da importância de se estudar as propriedades reológicas dos ligantes betuminosos e principalmente se forem modificados por polímeros Collins et al., 1991; Goodrich, 1991; Bahia et al., 1998, 1999). Em se tratando de um material termosensível como o asfalto, cuja consistência varia consideravelmente com a temperatura, é muito importante desenvolver relações entre o comportamento reológico e as condições ambientais.

O objetivo deste trabalho é determinar, através das propriedades reológicas, o parâmetro da energia de ativação de fluxo $\left(E_{f}\right)$ do ligante asfáltico puro e modificado por polímeros (SBS e EVA) e aditivos (cal hidratada, óleo extensor, e líquido da castanha de caju). Esta análise permite a avaliação das propriedades dos ligantes e os efeitos dos aditivos modificadores em relação à susceptibilidade térmica. Duas técnicas, viscosimetria e reometria, são utilizadas de modo a permitir 
o estabelecimento de uma correlação entre a $E_{f}$ e a susceptibilidade térmica.

\section{FUNDAMENTOS TEÓRICOS}

\subsection{Viscosidade}

Um material puramente viscoso é aquele que escoa e deforma irreversivelmente quando submetido a uma tensão cisalhante. A lei de Newton descreve o fluido ideal utilizando uma equação constitutiva na qual a tensão e a taxa de deformação estão relacionadas através de uma constante de proporcionalidade chamada viscosidade. Mais especificamente, a relação entre o tensor tensão e a taxa de deformação para fluidos puramente viscosos é dada conforme indica a Equação 1 (Bretas e D’Ávila, 2005):

$$
\tau_{i j}=\mu \times \dot{\gamma}_{i j}
$$

em que, $\tau_{i j}:$ tensor tensão;

$\mu$ : constante de proporcionalidade chamada de viscosidade Newtoniana ou simplesmente viscosidade;

$\dot{\gamma}_{i j}$ : taxa de cisalhamento.

Esse parâmetro $\mu$ representa a resistência ao fluxo ou ao escoamento do material. Quanto maior a viscosidade de um material, maior será a sua resistência ao escoamento. Fluidos que durante o escoamento possuem viscosidade constante são chamados de fluidos Newtonianos. Esses materiais apresentam a mesma resistência ao fluxo, independente das tensões ou deformações aplicadas. $\mathrm{O}$ asfalto é essencialmente um fluido Newtoniano e apresenta um valor de viscosidade independente da taxa de aplicação de carga.

Quando se tem taxas de cisalhamento intermediárias, a relação entre $\tau_{i j}$ e $\dot{\gamma}_{i j}$ não é uma constante e então essa relação é chamada de viscosidade nãoNewtoniana ou simplesmente $\eta$. Os fluidos que durante o escoamento apresentam esta relação entre a tensão e a taxa de cisalhamento são chamados de nãoNewtonianos. Os asfaltos modificados por polímeros comportam-se, dependendo da temperatura aplicada, como fluidos não-Newtonianos, apresentando características pseudoplásticas (Lu et al., 1999).

A Equação 1 também expressa uma característica importante dos fluidos puramente viscosos que é o fato de, ao se aplicar uma tensão, o fluido se deformar continuamente a uma determinada taxa $\mathrm{d} \gamma_{i j} / \mathrm{dt}$. Já os materiais sólidos, ao contrário, deformam-se a uma dada deformação $\gamma_{i j}$ ao ser aplicada uma tensão $\tau_{i j}$. Ou seja, a tensão de cisalhamento causa deformação nos sólidos, mas em fluidos ela causa uma taxa de deformação. Isso significa, simplesmente, que os sólidos podem ser elasticamente deformados, enquanto que os fluidos fluem (Schramm, 2006). Ligantes asfálticos são materiais com características viscoelásticas, ou seja, apresentam comportamento reológico tanto de sólidos elásticos como de fluidos viscosos.

Em regime oscilatório ou dinâmico de cisalhamento, o material em análise é submetido a uma tensão e/ou deformação oscilatória, e a resposta, deformação ou tensão correspondente, é medida. O comportamento reológico é medido em função do tempo, temperatura, tensão, deformação e freqüência. Materiais viscoelásticos, como, por exemplo, os ligantes asfálticos, apresentam tensão defasada em relação à deformação por um ângulo de defasagem $\delta$. A amplitude de tensão (ou a deformação) deverá ser pequena para garantir que as medidas estejam dentro do regime de viscoelasticidade linear. Neste caso, as tensões e/ou deformações estarão oscilando com a mesma freqüência $\omega$. A relação entre a tensão de cisalhamento e a deformação é o chamado módulo complexo $G^{*}$, conforme indica a Equação 2:

$$
G^{*}=\frac{\tau}{\gamma}
$$

sendo, $G^{*}:$ módulo complexo;

$$
\begin{aligned}
& \tau: \text { tensão; } \\
& \gamma: \text { deformação. }
\end{aligned}
$$

À semelhança dos fluidos puramente viscosos, pode-se definir a viscosidade complexa $\left(\eta^{*}\right)$, conforme indica a Equação 3:

$$
\eta^{*}=\frac{G^{*}}{\omega}=\frac{\tau_{\text {máx }}}{\gamma_{\text {máx }} \cdot \omega}
$$

sendo, $\eta^{*}$ : viscosidade complexa;

$G^{*}$ : módulo complexo;

$\omega$ : freqüência;

$\tau_{\text {máx }}$ : tensão máxima;

$\gamma_{\text {máx }}:$ deformação máxima.

A viscosidade complexa descreve a resistência total ao cisalhamento dinâmico, e pode ser segmentada em duas componentes, conforme as Equações 4 e 5.

$$
\begin{aligned}
& \eta^{\prime}=\frac{G^{\prime \prime}}{\omega}=\frac{\tau_{\text {máx }}}{\gamma_{\text {máx }} \cdot \omega} \cdot \operatorname{sen}(\delta) \\
& \eta^{\prime \prime}=\frac{G^{\prime}}{\omega}=\frac{\tau_{\text {máx }}}{\gamma_{\text {máx }} \cdot \omega} \cdot \cos (\delta)
\end{aligned}
$$

em que, $\eta^{\prime}$ : viscosidade de armazenamento, que seria a componente elástica;

$G^{\prime \prime}$ : módulo de perda;

$\tau_{\text {máx }}$ : tensão máxima;

$\gamma_{\text {máx }}:$ deformação máxima;

$\eta^{\prime \prime}$ : viscosidade dinâmica, que seria a componete viscosa;

$G^{\prime}$ : módulo de armazenamento. 


\subsection{Energia de ativação de fluxo}

O fluxo (escoamento) de um líquido pode ser considerado como um processo térmico em que as moléculas devem exceder uma barreira de energia para se movimentar. Quando se aumenta a temperatura, a energia térmica das moléculas também é aumentada, do mesmo modo como os sítios "vagos" existentes no fluido. Assim, quando um fluido escoa, são as camadas de moléculas que deslizam umas sobre as outras, para sobrepor-se às forças intermoleculares, que causam a resistência ao fluxo.

Tendo como base este modelo, e utilizando a técnica da viscosimetria, é possível estabelecer uma relação entre a viscosidade e a temperatura utilizando a equação de Arrhenius (Eying, 1936; Ward e Hadley, 1993; Painter e Coleman, 1997), conforme a Equação 6:

$$
\log (\eta)=\frac{E_{f}}{(R \cdot T)}+\ln \left(A_{t}\right)
$$

em que, $\eta$ : viscosidade do material;

$T$ : temperatura em graus Kelvin;

$A_{t}$ : fator pré-exponencial;

$E_{f}$ : energia de ativação do fluxo;

$R$ : constante universal dos gases $\left(8,314 \mathrm{~J} \cdot \mathrm{mol}^{-1} \cdot \mathrm{K}^{-1}\right)$.

Construindo um gráfico de $\ln \eta$ em função de 1/T, tem-se os valores da inclinação $\mathrm{E}_{\mathrm{f}} / \mathrm{R}$ de acordo com a Equação 7:

$$
\alpha=\frac{E_{f}}{R} \rightarrow E_{f}=\alpha \cdot R
$$

sendo, $\quad \alpha$ : coeficiente angular da reta;

$E_{f}$ : energia de ativação do fluxo;

$R$ : constante dos gases.

Utilizando-se a técnica da reometria, pode-se calcular o valor da energia de ativação de fluxo através do modelo de Arrhenius, conforme indica a Equação 8.

$$
\log \left(A_{t}\right)=\frac{E_{f}}{R \cdot\left(T-T_{0}\right)}
$$

sendo, $E_{f}$ : energia de ativação do fluxo associado com a relaxação;

$R$ : constante dos gases $\left(8,314 \mathrm{~J} \cdot \mathrm{mol}^{-1} \cdot \mathrm{K}^{-1}\right)$;

$T$ : temperatura medida;

$T_{0}:$ temperatura de referência;

$A_{t}$ : fator de deslocamento.

O método da superposição tempo-temperatura permite que se obtenham as curvas mestras das funções viscoelásticas linear (Partal et al., 1999). Os resultados de $G^{*}, G^{\prime}, G^{\prime \prime}$ e $\delta$, a uma dada temperatura, podem ser dispostos horizontalmente em uma escala log-log para se originar uma curva mestra (Polacco et al., 2003). Este procedimento permite prever as caracterís- ticas do ligante para faixas de freqüências específicas, que são de interesse técnico, porém, experimentalmente, difíceis de serem alcançadas.

O procedimento para determinação da curva mestra é baseado no princípio de superposição tempotemperatura (Pfeiffer e Van Doormal, 1936), que usa a equivalência entre freqüência e temperatura (Ferry, 1980), para o comportamento viscoelástico linear de asfaltos puros e modificados por polímeros. Parâmetros como $G^{*}, G^{\prime}, G^{\prime \prime}, \delta$ e $\eta^{*}$ são dispostos em um gráfico (escala log-log) em relação a freqüência, considerando diferentes temperaturas $\left(T_{1}, T_{2}, T_{3}, \ldots, T_{n}\right)$. Uma temperatura de referência é então escolhida e todas as outras temperaturas são transportadas horizontalmente, até que estas se ajustem na curva de referência. Para todas as curvas, nas temperaturas ensaiadas, têm-se associado um fator de deslocamento (ou conversão) $A_{t}$. Estes fatores de conversão são grafados em função da temperatura (superposição tempo-temperatura).

Os fatores do deslocamento podem ser relacionados aos valores dos parâmetros reológicos dos ligantes asfálticos, tais como viscosidade e o tempo médio de relaxação. A dependência do fator do deslocamento $\left(A_{t}\right)$ com a temperatura para o ligante asfáltico pode ser descrita pelas equações de Arrhenius ou WilliamsLandel-Ferry (WLF) (Williams et al., 1955; Dongré et al., 1996).

Os valores da energia de ativação de fluxo $\left(E_{f}\right)$ determinados pelas diferentes técnicas podem ser utilizados para estimar a susceptibilidade térmica dos ligantes asfálticos, assim como também podem ser usados para medir o esforço e a energia de compactação das misturas. Uma $E_{f}$ baixa indica que o ligante asfáltico tem menor sensibilidade, visto que uma energia de ativação de fluxo mais elevada mostra uma maior sensibilidade à temperatura (Coe e Cui, 2001).

\section{MATERIAIS E MÉTODOS}

\subsection{Materiais}

\subsubsection{Ligante asfáltico}

O ligante investigado é o CAP 50/70 processado na Lubnor/Petrobras por destilação a vácuo e oriundo do petróleo Fazenda Alegre, do estado do Espírito Santo. A amostra foi denominada CAPFA.

\subsubsection{Polímeros}

O copolímero SBS foi fornecido pela Petroflex Ind. e Com. S/A na forma de pellets. O copolímero EVA foi fornecido pela Politeno na forma de pellets, denominado Evateno HM 728, com teor de acetato de vinila de $28 \%(\mathrm{~m} / \mathrm{m})$. 


\subsubsection{Cal hidratada}

A cal foi fornecida pela empresa Carbomil na forma de pó, passante em uma peneira de 200 mesh.

\subsubsection{Líquido da castanha de caju}

O LCC foi proveniente da fábrica de castanha Cione (Fortaleza/CE).

\subsection{5. Óleo extensor}

Utilizou-se o óleo extensor PDAB/LPE/AsfaltoExtrato Aromático NP C698/00, cedido pelo Cenpes/Petrobras, para homogeneizar a mistura do ligante com o copolímero SBS.

\subsection{Métodos}

\subsubsection{Preparo das misturas}

As misturas foram preparadas utilizando-se um agitador de baixo cisalhamento, IKA modelo RW20, com agitação de $540 \pm 5 \mathrm{rpm}$ por um período de três (misturas com SBS) e duas (misturas com EVA) horas, na temperatura de $160 \pm 5{ }^{\circ} \mathrm{C}$. O misturador é equipado com controle de temperatura, agitador mecânico de baixo cisalhamento e hélice cisalhante.

As misturas de CAP modificado pelo polímero SBS foram feitas com o CAPFA e 4,5\% em peso de SBS. Outra mistura foi realizada adicionando-se $1,5 \% \mathrm{em}$ peso do diluente aromático como agente compatibilizante. As amostras foram denominadas CAPFA $+4,5 \%$ SBS e CAPFA $+4,5 \%$ SBS $+1,5 \%$ OE. O CAPFA também foi modificado pelo polímero EVA, utilizando-se 4 e $6 \%$ em peso do polímero, e pelos aditivos cal hidratada e líquido da castanha de caju (LCC), utilizando-se $2 \%$ em peso de cada aditivo. Desse modo, três misturas foram produzidas: CAPFA $+6 \%$ EVA, $\quad$ CAPFA $+4 \%$ EVA $+2 \% \mathrm{CAL}$ e CAPFA $+4 \%$ EVA $+2 \%$ LCC.

\subsubsection{Envelhecimento oxidativo em estufa de filme fino rotativo}

O CAP puro e modificado foi envelhecido usando uma estufa de filme fino rotativo (Rolling Thin Film Oven Test - RTFOT) (fabricante DESPATCH), conforme a ASTM D2872 (1997). Este método de teste indica mudanças nas propriedades do asfalto que podem ocorrer durante a usinagem. Em recipientes de vidros foram pesadas aproximadamente $35 \mathrm{~g}$ de amostra. As amostras foram aquecidas a $163^{\circ} \mathrm{C}$ por $85 \mathrm{mi}-$ nutos e resfriadas à temperatura ambiente. As alterações reológicas foram verificadas por meio de ensaios dinâmicos-mecânicos e de viscosidade.

\subsubsection{Viscosidade}

A viscosidade absoluta foi determinada conforme a ASTM D4402 (1987) nas seguintes temperaturas: 135,150 , e $175^{\circ} \mathrm{C}$ a diferentes taxas de cisalhamento, utilizando-se o viscosímetro rotacional cilíndrico Bro- okfield modelo DVII ${ }^{+}$acoplado a um controlador de temperatura THERMOSEL. A viscosidade é medida através do torque necessário para rodar uma haste de prova (spindle) imersa na amostra de asfalto quente. Utilizou-se uma rotação de $20 \mathrm{rpm}$ e spindles 21 e 27, respectivamente, para o CAP e para o CAP modificado pelos polímeros e aditivos. Utilizando-se a dependência da viscosidade em função da temperatura, a energia de ativação de fluxo foi calculada a partir da equação de Arrhenius, conforme descrito no item 2.2.

\subsubsection{Ensaios dinâmicos-mecânicos}

Estes ensaios foram realizados utilizando-se o reômetro de cisalhamento dinâmico (DSR) modelo AR 2000 (TA Instruments). Em um molde de silicone foram preparados os corpos de prova de aproximadamente $1 \mathrm{~mm}$ de espessura e $25 \mathrm{~mm}$ de diâmetro e em seguida estes foram testados em spindles de placas paralelas com diâmetro de $25 \mathrm{~mm}$. Os resultados de $G^{*}$ e $\delta$, a uma temperatura de referência em $25^{\circ} \mathrm{C}$ (frequency sweep de 0,01 a $10 \mathrm{~Hz}$ e de 15 a $65^{\circ} \mathrm{C}$ ), foram dispostos horizontalmente em uma escala log-log para originar a curva mestra. A partir desta construção calculouse a energia de ativação de fluxo $\left(E_{f}\right)$ das amostras, utilizando-se do procedimento já descrito no item 2.2.

\section{RESULTADOS E DISCUSSÃO}

\subsection{Energia de ativação de fluxo $\left(E_{f}\right)$ por Viscosimetria}

A faixa de temperatura utilizada foi de 135 a $175^{\circ} \mathrm{C}$, visto que, na prática, a temperatura utilizada nos processos de mistura e compactação, está entre 145 e $165^{\circ} \mathrm{C}$. As Figuras 1 e 2 mostram, respectivamente, o gráfico de Arrhenius obtido antes e após o envelhecimento RTFOT para o ligante asfáltico puro e modificado.

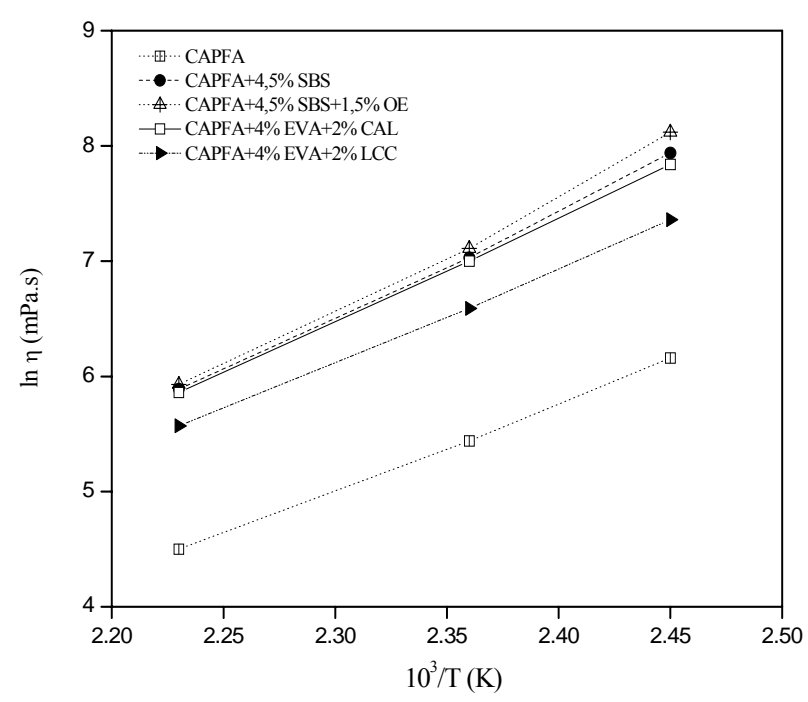

Figura 1. Gráfico de Arrhenius para CAPFA puro e modificado 


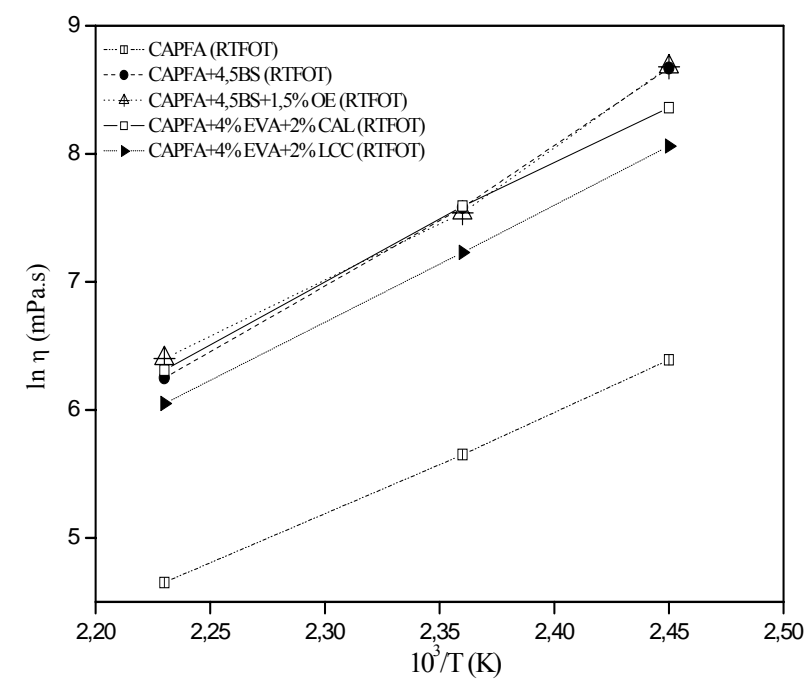

Figura 2. Gráfico de Arrhenius após RTFOT para CAPFA puro e modificado

A Energia de Ativação de Fluxo $\left(E_{f}\right)$ foi calculada multiplicando-se a inclinação da reta pela constante universal dos gases $(R)$, conforme relação descrita na Equação 7. A Tabela 1 apresenta os valores da $E_{f}$ encontrados para cada mistura (CAP - polímero - aditivo).

Tabela 1. Valores da $E_{f}$ na faixa de temperatura entre $135 \mathrm{e}$ $175^{\circ} \mathrm{C}$, antes e após o envelhecimento RTFOT

\begin{tabular}{lcc}
\multicolumn{1}{c}{ Amostras } & $\begin{array}{c}\text { Antes do } \\
\text { RTFOT } \\
\boldsymbol{E}_{f}(\mathbf{k J} / \mathbf{m o l})\end{array}$ & $\begin{array}{c}\text { Após } \\
\boldsymbol{R T F O T} \\
\boldsymbol{E}_{f}(\mathbf{k J} / \mathbf{m o l})\end{array}$ \\
\hline CAPFA & 63 & 66 \\
\hline CAPFA+4\%EVA+2\%LCC & 67 & 76 \\
\hline CAPFA+4\%EVA+2\%CAL & 75 & 78 \\
\hline CAPFA+4,5\%SBS & 77 & 91 \\
\hline CAPFA+4,5\% SBS+1,5\%OE & 82 & 85 \\
\hline
\end{tabular}

Os resultados indicam diferentes valores de energia de ativação de fluxo para o ligante asfáltico puro e modificado por polímeros e aditivos. Devido à modificação do CAPFA pelos polímeros, obtiveram-se maiores valores da $E_{f}$ para as misturas do que para o ligante original. A justificativa para este comportamento se deve ao fato de que o tipo e o teor de polímero utilizado têm influência sobre a energia de ativação de fluxo do ligante (Coe e Cui, 2001).

Observa-se também que o ligante modificado pelo polímero SBS apresentou valores de $E_{f}$ maiores do que quando modificado pelo polímero EVA. Sabendo que uma Energia de Ativação de Fluxo baixa indica que o ligante asfáltico é menos sensível a variação de temperatura, e que uma $E_{f}$ mais alta indica uma maior sensibilidade (Coe e Cui, 2001), tem-se a seguinte ordem em relação à susceptibilidade térmica para as misturas asfálticas antes do envelhecimento RTFOT:

CAPFA $+4 \%$ EVA $+2 \%$ LCC $<$

CAPFA $+4 \%$ EVA $+2 \% \mathrm{CAL}<$

$\mathrm{CAPFA}+4,5 \% \mathrm{SBS}<\mathrm{CAPFA}+4,5 \% \mathrm{SBS}+1,5 \% \mathrm{OE}$.
Verifica-se também, de acordo com os valores da Tabela 1, que o envelhecimento oxidativo aumentou a energia de ativação de fluxo do ligante. Este incremento pode ser explicado pelo fato de que a oxidação ocasiona um aumento na quantidade de moléculas polares presentes no ligante asfáltico, e uma concentração elevada dessas moléculas proporciona um aumento das forças intermoleculares que por sua vez conduzem a interações mais fortes. Estas interações dentro do ligante resultam em uma maior resistência ao fluxo e, por conseqüência, uma maior barreira da Energia de Ativação de Fluxo. Assim, a susceptibilidade relativa à temperatura das misturas asfálticas envelhecidas apresenta a seguinte ordem:

$\mathrm{CAPFA}+4 \% \mathrm{EVA}+2 \% \mathrm{LCC}<$

$\mathrm{CAPFA}+4 \% \mathrm{EVA}+2 \% \mathrm{CAL}<$

$\mathrm{CAPFA}+4,5 \% \mathrm{SBS}+1,5 \% \mathrm{OE}<\mathrm{CAPFA}+4,5 \% \mathrm{SBS}$.

Observa-se que as misturas contendo EVA se degradam menos do que aquelas que contêm SBS, uma vez que no polímero EVA não existem duplas ligações butadiênicas que se oxidam facilmente, como no caso do SBS. É necessário também considerar o efeito positivo dos aditivos presentes nas misturas contendo o polímero EVA, uma vez que a cal hidratada, por suas características surfactantes, tem a habilidade de reduzir o envelhecimento da mistura asfáltica através da interação com compostos polares reativos presentes no ligante e o LCC, por possuir grupos fenólicos, agir como um excelente antioxidante.

\subsection{Energia de ativação de fluxo $\left(E_{f}\right)$ por Reometria}

Os fatores de deslocamento $\left(A_{t}\right)$ foram determinados experimentalmente através das curvas mestras utilizando a temperatura de $25^{\circ} \mathrm{C}$ como a de referência, os fatores de deslocamento $\left(A_{t}\right)$ foram determinados experimentalmente através das curvas mestras. Em seguida, aplicando-se a equação WLF, conforme a Equação 7, calculou-se a energia de ativação de fluxo das amostras, antes e após RTFOT. Estes valores estão listados nas Tabelas 2 e 3.

De acordo com a Tabela 2, verifica-se que os valores da energia de ativação de fluxo obtidos para o CAPFA+4,5\%SBS não envelhecido são mais elevados

Tabela 2. $E_{f}(\mathrm{~kJ} / \mathrm{mol})$ das amostras

\begin{tabular}{cccc}
\hline & \multicolumn{3}{c}{$\boldsymbol{E}_{\boldsymbol{f}}(\mathbf{k J} / \mathbf{m o l})$} \\
\cline { 2 - 4 } $\boldsymbol{T}\left({ }^{\circ} \mathrm{C}\right)$ & CAPFA & $\begin{array}{c}\text { CAPFA+ } \\
\mathbf{4 , 5 \% S B S}\end{array}$ & $\begin{array}{c}\text { CAPFA+ } \\
\mathbf{4 \% E V A +} \\
\mathbf{2 \% C A L}\end{array}$ \\
\hline 40 & 16 & 19 & 17 \\
\hline 45 & 42 & 50 & 39 \\
\hline 50 & 71 & 80 & 67 \\
\hline 55 & 102 & 113 & 98 \\
\hline 60 & 134 & 150 & 132 \\
\hline 65 & 170 & 187 & 178 \\
\hline
\end{tabular}


do que aqueles encontrados para o CAP puro e para a amostra $\mathrm{CAPFA}+4 \% \mathrm{EVA}+2 \% \mathrm{CAL}$, embora não haja diferenças significativas entre os valores do CAPFA e aqueles encontrados para o ligante asfáltico modificado com EVA. A susceptibilidade à temperatura da amostra CAPFA $+4,5 \%$ SBS foi, portanto, mais elevada do que para o ligante não modificado CAPFA e modificado com EVA. O CAPFA e as misturas CAPFA $+4,5 \%$ SBS e CAPFA $+4 \% E V A+2 \% \mathrm{CAL}$ apresentaram valores de energia de ativação de fluxo muito semelhantes aos valores encontrados na literatura obtidos de outros estudos (Verney et al., 1990; Partal et al., 1999), com valores de $E_{f}=149 \mathrm{~kJ} / \mathrm{mol}$ acima de $40{ }^{\circ} \mathrm{C}$.

$\mathrm{Na}$ faixa de temperatura ensaiada $\left(40\right.$ a $\left.65{ }^{\circ} \mathrm{C}\right)$, os dados obtidos podem ser correlacionados com a susceptibilidade térmica dos ligantes ao longo da vida útil do pavimento, mais precisamente, em regiões onde as temperaturas são mais elevadas.

Após o envelhecimento simulado RTFOT, como esperado, observa-se que a amostra envelhecida do ligante CAPFA apresenta valores de $E_{f}$ maiores (Tabela 3) do que a amostra não-envelhecida. Como mencionado anteriormente, o processo oxidativo causa o endurecimento do ligante, que provêm da ocorrência de interações mais fortes entre os componentes do asfalto (Ruan et al., 2003), resultando também em um aumento da $E_{f .}$.

Tabela 3. $E_{f}(\mathrm{~kJ} / \mathrm{mol})$ das amostras após RTFOT

\begin{tabular}{cccc}
\hline & \multicolumn{3}{c}{$\boldsymbol{E}_{\boldsymbol{f}}(\mathbf{k J} / \mathbf{m o l})$} \\
\cline { 2 - 4 } $\boldsymbol{T}\left({ }^{\circ} \mathrm{C}\right)$ & CAPFA & $\begin{array}{c}\text { CAPFA+ } \\
\mathbf{4 , 5 \% S B S}\end{array}$ & $\begin{array}{c}\text { CAPFA+ } \\
\mathbf{4 \% E V A +} \\
\mathbf{2 \% C A L}\end{array}$ \\
\hline 40 & 17 & 21 & 16 \\
\hline 45 & 45 & 53 & 51 \\
\hline 50 & 75 & 81 & 80 \\
\hline 55 & 105 & 114 & 111 \\
\hline 60 & 137 & 148 & 146 \\
\hline 65 & 173 & 185 & 170 \\
\hline
\end{tabular}

No entanto, para o ligante asfáltico modificado por polímeros essa tendência foi oposta nas temperaturas mais altas. Ruan et al. (2003) sugerem uma explicação para este fato: é que a oxidação degrada as longas cadeias moleculares do polímero em cadeias mais curtas e de menor dimensão, e isto irá reduzir as interações entre os componentes do polímero e do ligante. Desse modo, a energia de ativação de fluxo para o sistema asfalto-polímero diminui com a oxidação.

\section{CONCLUSÕES}

Utilizando-se das técnicas de viscosimetria e reometria foi possível avaliar a susceptibilidade térmica dos ligantes asfálticos puros e modificados por polímeros e aditivos, por meio do cálculo da energia de ativação de fluxo. Os valores obtidos permitiram estabelecer uma análise comparativa da susceptibilidade térmica dos ligantes. As misturas contendo EVA apresentaram menor $\mathrm{E}_{\mathrm{f}} \mathrm{e}$, consequentemente, menor susceptibilidade térmica quando comparadas às misturas contendo SBS, independentemente da técnica utilizada e da faixa de temperatura ensaiada. Os aditivos presentes nas misturas contendo o polímero EVA também contribuíram para a menor sensibilidade às variações de temperatura da amostra. A cal hidratada contribuiu para reduzir a $E_{f}$ através da interação com grupos polares presentes no ligante. O LCC, por sua vez, devido a sua estrutura e propriedade antioxidante, provavelmente, influiu de modo a reduzir a barreira energética necessária ao fluxo do ligante.

\section{AGRADECIMENTOS}

Os autores agradecem a Lubnor/Petrobras, pela doação do CAP 50/70 oriundo do petróleo Fazenda Alegre; a Petroflex Ind. e Com. S/A, pelo copolímero SBS fornecido; a Politeno, pelo fornecimento do copolímero EVA; e a Carbomil, pela cal hidratada fornecida. Raquel M. Bringel agradece à ANP pela bolsa concedida.

\section{REFERÊNCIAS BIBLIOGRÁFICAS}

Alencar, A. E. V.; Bringel, R. M.; Lucena, M. C. C.; Soares, J. B.; Soares, S. A. (2006) Efeito da Presença de Aditivos nas Propriedades Reológicas do Ligante Asfaltico Modificado por EVA. Instituto Brasileiro de Petróleo e Gás - IBP. $18^{\circ}$ Encontro de Asfalto, Rio de Janeiro/RJ, cd-room IBP0035_06, p. 28.

Alencar, A. E. V.; Bringel, R. M.; Soares, J. B.; Soares, S. A. (2007) Rheological Behavior of Asphalt Binder Modified with EVA Copolymer and Coconut Fiber. XI International Macromolecular Colloquium and the $6^{\text {th }}$ International Symposium on Natural Polymers and Composites - Isnapol, Gramado/RS.

ASTM (1987) D4402 - Standard Test Method for Viscosity Determinations of Unfilled Asphalts Using the Brookfield Thermosel Apparatus. American Society for Testing and Materials.

ASTM (1997) D2872 - Standard Test Method for Effect of Heat and Air on a Moving Film of Asphalt (Rolling Thin-Film Oven Test). American Society for Testing and Materials.

Bahia, H. U.; Hislop, W. P.; Zhai, H.; Rangel, A. (1998) Classification of Asphalt Binders Into Simple and Complex Binders. Journal of the Association of Asphalt Paving Technologists, v. 67, p. 1-41.

Bahia, H. U.; Zhai, H.; Bonnetti, K.; Kose, S. (1999) Non-Linear Viscoelastic and Fatigue Properties of Asphalt Binders. Journal of the Association of Asphalt Paving Technologists, v. 68, p. 1-34.

Bretas, R. E. S.; D’Ávila, M. A. (2005) Reologia de Polímeros Fundidos ( $2^{\circ}$ ed.). EdUFSCar, São Carlos.

Bringel, R. M. (2007) Estudo Químico e Reológico de Ligantes Asfálticos Modificados por Polímeros e Aditivos. Tese de Mestrado, Universidade Federal do Ceará, Fortaleza/CE.

Bringel, R. M.; Alencar, A. E. V.; Lucena, M. C. C.; Soares, J. B.; Soares, S. A. (2006) Comportamento Reológico do Ligante Asfáltico Modificado por SBS e Diluente Aromático como Compatibilizante. Instituto Brasileiro de Petróleo e Gás - IBP. $18^{\circ}$ Encontro de Asfalto, Rio de Janeiro/RJ, cd-rom IBP0033_06, p. 27.

Bringel, R. M.; Soares, J. B.; Soares, S. A. (2005) Propriedades Químicas e Reológicas de Asfalto Modificado por Copolímero EVA. $3^{\circ} \mathrm{Con}$ gresso Brasileiro de P\&D em Petróleo e Gás, Salvador/BA, p. 697-698.

Coe, D. S.; Cui, H. Z. (2001) Determinación de la Energia de Activación de Flujo en Ligantes Asfálticos. Idaho Asphalt Supply, Inc. Nampa, Idaho, USA, p. 10.

Collins, J. H.; Bouldin, M. G.; Gelles, R.; Berker, A. (1991) Improved Performance of Paving Asphalts by Polymer Modification. Journal of the Association of Asphalt Paving Technologists, v. 60, p. 4379.

Dongré, R.; Youtcheff, J.; Anderson, D. (1996) Better Roads Through Rheology. Applied Rheology, v. 6, p. 75-82. 
Eyring, H. (1936) Viscosity, Plasticity, and Diffusion as Examples of Absolute Reaction Rates. Journal of Chemical Physics, v. 4, p. $283-$ 291.

Ferry, J. D. (1980) Viscoelastic Properties of Polymers ( $3^{\circ}$ ed.). John Wiley \& Sons, New York.

Gonzalez, O.; Muñoz, M. E.; Santamaría, A.; García-Morales, M.; Navarro, F. J.; Partal, P. (2004) Rheology and Stability of Bitumen/EVA Blends. European Polymer Journal, p. 2365-2372.

Goodrich, J. L. (1991) Asphalt Binder Rheology, Asphalt Concrete Rheology and Asphalt Concrete Mix Properties. Journal of the Association of Asphalt Paving Technologists, v. 60, p. 80-120.

Jin, H.; Gao, G.; Zhang, Y.; Zhang, Y.; Sun, K.; Fan, Y. (2002) Improved Properties of Polystyrene-Modified Asphalt Through Dynamic Vulcanization. Polymer Testing, v. 21, p. 633-640.

Lu, X.; Isacsson, U.; Ekblad, J. (1999) Rheological Properties of SBS, EVA and EBA Polymer Modified Bitumens. Materials and Structures, v. 32, p. 131-139.

Lucena, M. C. C. (2005) Caracterização Química e Reológica de Asfaltos Modificados por Polímeros. Tese de Doutorado, Universidade Federal do Ceará, Fortaleza/CE.

Masson, J. F.; Perc, S. B. (2005) Solventeless Fingerprinting of Bituminous Materials: A High-Resolution Thermogravimetric Method. Thermoquimica Acta, v. 436, p. 35-42.

Painter, P. C.; Coleman, M. M. (1997) Fundamentals of Polymer ScienceAn Introductory Test. Technomic Publishing Co. Inc., Lancaster, PA.

Partal, P.; Martínez-Boza, F.; Conde, B.; Gallegos, C. (1999) Rheological Characterisation of Synthetic Binders and Unmodified Bitumens. Fuel, v. 78, p. 1-10.

Pfeiffer, J. P.; Van Doormal, P. M. (1936) The Rheological Properties of Asphaltic Bitumens. Journal Institute of Petroleum, v. 22, p. 414440.

Pinto, S. (1991) Estudo do Comportamento à Fadiga de Misturas Betuminosas e Aplicação na Avaliação Estrutural de Pavimentos. Tese de Doutorado, Universidade Federal do Rio de Janeiro, Rio de Janeiro/RJ

Polacco, G.; Vacin, O. J.; Biondi, D.; Stastna, J.; Zanzotto, L. (2003) Dynamic Master Curve of Polymer Modified Asphalt from Three Different Geometries. Applied Rheology, v. 13, p. 118-124.

Ruan, Y.; Davison, R. R.; Glover, C. J. (2003) The Effect of Long-Term Oxidation on the Rheological Properties of Polymer Modified Asphalts. Fuel, v. 82, p. 1763-1773.

Schramm, G. (2006) Reologia e Reometria: Fundamentos Teóricos e Práticos ( $2^{\circ}$ ed.). Tradução e Adaptação: Cheila G. Mothé; Denise Z. Correia; Hans M. Petri; Michelle Gonçalves; Tatiana Carestiano. Artliber Editora, São Paulo, 2006.

Verney, V.; Michel, A.; Planche, J-P; Brulé, B. (1990) Influence de la Température Sur la Viscosité Complex D’un Bitume Routier. Proceedings Rhéologie des Matériaux du Génie Civil (25 éme colloque du GFR), Grenoble, France, p. 257-268.

Ward, I. M.; Hadley, D. W. (1993) An Introduction to the Mechanical Properties of Solid Polymers. Wiley, New York.

Williams, M. L; Landel, R. F; Ferry, J. D. (1955) The Temperature Dependence of The Relaxation Mechanisms in Amorphous Polymers and Other Glass Forming Liquids. Journal of the American Chemical Society, v. 77, p. 3701-3707.

Yildirim, Y. (2007) Polymer Modified Asphalt Binders. Construction and Building Materials, v. 21, p. 66-72. 